\title{
Perinatal care in a patient with diagnosed Westphal's disease
}

\author{
Marek Szyszkowski ${ }^{1}$, Dominik Pruski ${ }^{1}$, Paulina Banach ${ }^{2}$, Marcin Przybylski ${ }^{1}$ \\ ${ }^{1}$ Department of Obstetrics and Gynecology, Dictrict Public Hospital in Poznan, Poland \\ ${ }^{2}$ Gynecologic Oncology Department, Poznan University of Medical Sciences, Poznan, Poland
}

Hypokalemic periodic paralysis (HOKPP) belongs to the group of genetically conditioned myopathies, inherited in an autosomal dominant manner. Pathology is associated with a fall in blood potassium levels (hypokalemia). The disorder is linked to the region of chromosome 1q 31-32 in the gene encoding the dihydropyridine (DHP) receptor and the unit of the alpha-1 calcium channel. Impaired function of ion channels and sodium-potassium pump lead to secondary depolarization of cell membranes and further lack of muscle reaction to nervous stimulation. The Westphal's disease is characterized by the occurrence of quadriplegic paralysis associated with hypokalemia. In the severe form of the disease, a generalized paralysis may occur with involvement of respiratory muscles and arrhythmias. The factors causing the seizure include: sudden temperature changes, cold, long-term immobility, vigorous physical exercise, stress, viral infections. Prevention consists of avoiding risk factors and treatment based on the supply of potassium preparations and the use of mechanical lung ventilation.

\section{CASE REPORT}

A 27-year-old female patient came to the hospital, at 41st week pregnant with the first child to induce labor. The course of pregnancy was uncomplicated. The patient did not report any symptoms at the time. Patient's medical history included Westphal's disease, diagnosed in her childhood. She inherited a pregnant illness from her father who has been ill since he was 12 years old. Since the first episode in the third year of life, the patient was under constant care of the Neurological Clinic, hospitalized several times and treated conservatively with potassium preparations. The patient admitted to being supplied with potassium at time of hospital admission.

Due to the 41st week of pregnancy, the decision was made to insert Foley catheter to pre-induce, then to provide oxytocin for labor induction. Before pre-induction, the potassium level blood test was carried, measuring $4.00 \mathrm{mmol} / \mathrm{L}$. During the procedure, the patient reported numbness in the limbs, which according to previous experience of the patient could suggest a decrease in potassium concentration. An ECG was performed in which no disturbances were found. Blood tests showed hypokalaemia at $3.2 \mathrm{mmol} / \mathrm{L} .20 \mathrm{mEq} \mathrm{KCl}$ was given intravenously as a result of which the symptoms disappeared. After a few hours of induction, amniotomy and administration of diastolic drugs (buskolysin) decelerations occurred in the CTG record. Due to the risk of fetus life, the pregnancy was terminated by caesarean section. A son with a mass of $3400 \mathrm{~g} \mathrm{Ap} 10$ was born. In the puerperium period, potassium was supplemented by monitoring its concentration. During the hospitalization period, the patient did not report any recurrence of neurological symptoms. On the fifth day after delivery, she was discharged home with recommendations for neurological control in an outpatient procedure and urgent report to the hospital in case of alarming symptoms. 